\title{
Study on the Effect of Green's Education Model in Improving the Knowledge, Attitude and Practice of Occupational Protection in Chemotherapy among Nursing Interns
}

\author{
Shuzhi Xue*, Li He, Min Zhang \\ Medical Oncology Ward, Shaanxi Provincial People’s Hospital, Xi’an, Shaanxi, China.
}

How to cite this paper: Shuzhi Xue, $\mathrm{Li}$ He, Min Zhang. (2021) Study on the Effect of Green's Education Model in Improving the Knowledge, Attitude and Practice of Occupational Protection in Chemotherapy among Nursing Interns. International Journal of Clinical and Experimental Medicine Research, 5(4), 445-450. DOI: 10.26855/ijcemr.2021.10.004

Received: August 11, 2021

Accepted: September 4, 2021

Published: September 14, 2021

*Corresponding author: Shuzhi Xue, Medical Oncology Ward, Shaanxi Provincial People's Hospital, Xi'an, Shaanxi, China.

\begin{abstract}
Objective: To study the effect of green education model in improving the knowledge, attitude and practice of occupational protection in chemotherapy among nursing interns. Methods: 100 nursing interns in our hospital from February 2020 to February 2021 were selected retrospectively. According to the method of occupational protection health education, they were divided into two groups: systematic occupational protection health education group under the guidance of green education model (system group) and traditional occupational protection health education group under the guidance of green education model (traditional group), 50 cases in each group, the interns before and after the practice of chemotherapy occupational protection knowledge-attitude-practice, chemotherapy occupational exposure of the two groups were statistically analyzed. Results: The scores of knowledge, attitude and behavior of the two groups after internship were higher than those before internship $(P<0.05)$; after practice, the scores of knowledge, attitude and behavior of the system group were higher than those of the traditional group $(P<$ 0.05 ). The occupational exposure scores of the two groups after internship were lower than those before internship $(P<0.05)$; after practice, the occupational exposure score of the system group was lower than that of the traditional group $(P<0.05)$. Conclusion: Green education model has a good effect in improving nursing interns' knowledge, attitude and practice of occupational protection in chemotherapy.
\end{abstract}

\section{Keywords}

Green Education Model, Nursing Interns, Chemotherapy, Occupational Protection, Knowledge, Belief And Practice, Occupational Exposure

As a special group in the nursing team, the probabilities of occupational exposure of nursing students increase because of their weak awareness of occupational protection and poor compliance. The survey showed that the awareness rates of occupational exposure, post-treatment of occupational exposure and emergency plan of sharp instrument injury were $28.93 \%, 13.71 \%$ and $29.95 \%$ respectively, and $76.10 \%$ of nursing students had needle stick injury during the internship [1]. Health education intervention is the most effective way to reduce the occupational exposure of nursing students. The precede proceed health education model, founded by American health educationist green and others, emphasizes the intervention of belief and behavior while paying attention to knowledge sup- 
plement, which provides evidence-based basis and theoretical framework for the research of health education intervention [2]. This paper studies the effect of green education model in improving the knowledge, attitude and practice of occupational protection of nursing interns in chemotherapy.

\section{Materials and methods}

\subsection{General information}

100 nursing interns in our hospital from February 2020 to February 2021 were selected retrospectively. According to the methods of occupational protection health education, 100 nursing interns were divided into two groups: the system group and the traditional occupational protection health education group (traditional group) under the guidance of green education mode. The average age of 50 interns in the system group was 19-24 years (21.35 years) \pm The age of 1.62 was $48(96.00 \%)$ and $2(4.00 \%)$ were male. In the aspect of the grade of the internship hospital, 11 cases $(22.00 \%), 19$ cases $(38.00 \%)$ were at the second level and $20(40.00 \%)$ at the third level; in terms of vocational protection training, $10(20.00 \%)$ and $40(80.00 \%)$ were not participated in; in the study hospital, 46 patients (92.00\%), 3 uncertain (6.00\%) and $1(2.00 \%)$ were not willing. The average age of 50 interns in traditional group was $18-23$ years (20.35) \pm The age of 1.56 was $49(98.00 \%)$ and that of male was $49(98.00 \%)$, and $1(2.00 \%)$ was male. In the aspect of the grade of the internship hospital, 12 cases $(24.00 \%), 18$ cases $(36.00 \%)$ were in the second level and $20(40.00 \%)$ in the third level; In terms of vocational protection training, $11(22.00 \%)$ and 39 (78.00\%) were not involved; In the study hospital, 45 patients (90.00\%), 3 uncertain $(6.00 \%)$ and $2(4.00 \%)$ were not willing. The general data of the two groups were not significantly different $(P>0.05)$.

\subsection{Methods}

\subsubsection{Traditional group}

The occupation survey and self-protection knowledge, attitude and practice of two groups of nursing students were investigated in May 2012 by self-made questionnaire on the exposure and protection of occupational hazards during nursing students' occupation during the internship period. It was found that the exposure of chemical occupation hazards to nursing students during the internship period was very common. Disinfectants, antibiotics, anesthetic gases and antineoplastic drugs were the most frequently exposed; the exposure of psychosocial occupational hazards cannot be ignored, mainly for the night shift and work pressure exposure; sharp instrument injury and occupational exposure of blood and body fluid should be paid more attention; however, nursing students did not pay enough attention to the exposure of physical occupational hazards; the cognition of occupational protection related knowledge was lack in different degrees, the attitude of nursing students was relatively positive, and the behavior of occupational protection was poor. Before the internship, they received the traditional health education lecture on "occupational exposure risk factors and protection of medical staff”, which was taught by PPT and played by video for 3 class hours.

\subsubsection{System group}

(1) Develop and implement health education intervention program

The tendency factors of occupational protection behavior include the attitude, belief, values and cognition of nursing students, which are the motivation to promote or prevent their occupational protection behaviors. In view of the tendency factors, the group made health education intervention plan based on the evaluation results of the preliminary baseline survey and the needs of vocational protection education for nursing students, taking green health education model as the theoretical framework. According to the intervention plan, the nursing students in green group were systematically intervened in occupational protection health education one week before the internship, including the education of occupational protection attitude, belief, knowledge, skills and values. That is, Week 1 : use teaching and PPT demonstration training methods, train the relevant concepts and classification of occupational exposure ( 1 class hour), and the policies and regulations related to nursing occupational protection (1 class hour), use the training methods of scenario simulation and operation demonstration, and training standard preventive measures: hand washing and hand detoxification, wearing gloves, masks and goggles, wearing a separate clothing (1 class hour); Week 2: training the protection of blood source occupational exposure and sharp instrument injury by teaching, PPT demonstration and case analysis training methods: hazard factors, hazard modes or high-risk operation, protection methods and treatment after hazard occurrence (2 class hours), and training safety injection equipment and sharper box by using scenario simulation and demonstration operation training methods, Use and treatment of disposable medical supplies (1 class hour); Week 3: training chemotherapy drugs protection by teaching, PPT demonstration and case analysis training: operation protection, accidental exposure treatment, pollutant 
treatment and waste treatment (2 class hours), chemical disinfectant, antibiotics and other chemical occupational hazards (1 class hour); Week 4: training professional musculoskeletal disease protection by teaching and PPT demonstration training: nursing patients with mechanical principles, such as correct lifting technology, correct use of various auxiliary devices and increasing muscle strength and elasticity (1 class hour), using scenario simulation and demonstration operation training methods, training elastic socks The use of lumbar support and handling board (1 class hour), the teaching, PPT demonstration, case analysis training methods were used to train the prevention and active response of work stress and workplace violence (1 class hour).

(2) Strengthen the management of the intern nursing students

During the internship, the researchers provide further guidance and encourage nursing students to keep in touch during the internship. The specific contents include: (a) establishing the information platform for nursing students: researchers should establish nursing students' files before the experimental group nurses enter clinical practice, improve their personal information, especially mobile phone contact number, and establish Feixin group, so as to release information and manage them in a unified manner in time and keep in touch. Set up correspondent according to hospital. And create QQ group, which is convenient for nursing students to discuss each other during internship. Researchers join the QQ group to solve various questions of students. (b) The first month of internship is the key period to adapt to the environment and standardize nursing behaviors. Researchers contact the correspondents every week to understand the internship situation of students, urge them to report the internship safety accidents and provide guidance for the corresponding problems. At the end of the first month, the researcher personally contacted the nursing students in the experimental group for about 30min. The specific understanding of the occupational protection and questions during the internship was made, and timely answers were given, such as vaccination, whether occupational exposure occurred, occupational exposure, and how to prevent later. The problems encountered by nursing students during the internship can be timely feedback and guidance. (c) Within 3 months of practice, the researchers used the opportunity of sorting out nursing assessment (our school conducted overall nursing assessment for the intern nursing students every month), and conducted one overall nursing assessment for each nursing student in batches. The nurse shall be informed one week in advance to select the cases that have been nursing in clinical. The teacher shall ask the nursing students one-on-one questions about the case, and assess one nursing operation. During the operation assessment, observe the occupational protection behavior of the nursing students, strengthen the positive through praise, give targeted guidance to the weak links, and at the same time, the occupational exposure and protection status during the internship of the nursing students, For example, what confusion exists in occupational protection of nursing students, etc., provide timely guidance for $1 \mathrm{H}$. (d) In the third month of internship, the nursing students in the experimental group were concentrated by taking the opportunity of the first graduation examination to analyze the occupational exposure events occurred by the students in the three months, for about 2 hours, so that the nursing students can learn lessons, be alert to the students of occupational exposure events and improve their awareness and ability of occupational protection.

\subsection{Observation index}

The patients were followed up for 1 month. (1) Self protection knowledge, attitude and behavior questionnaire: the knowledge part is composed of 15 items, and 1 point is obtained if it is selected correctly, and the highest score is 15 points; in the attitude part, there are 30 items, which are scored by Likert 5, with the lowest score of 30 and the highest score of 150 . The higher the score is, the more positive the occupational protection attitude is; there are 36 items in the behavior part, and the Likert 5-level scoring method is also used, with the lowest score of 36 and the highest score of 180 . The higher the score is, the better the occupational protection behavior is. The total content validity coefficient (CVI) of the questionnaire was 0.852; the total Cronbach's score was obtained after pre-survey of 30 nursing students, $\alpha$ the coefficient is 0.726 . Half a month later, the same group of nursing students was asked to fill in the questionnaire again, and the total correlation coefficient was 0.862 [3]; (2) Occupational exposure questionnaire: divided into biological factors (8 items), chemical factors (10 items), physical factors (15 items) and psychosocial factors (2 factors), a total of 35 items. The highest score was 115 and the lowest was 18. The higher the value, the more serious the occupational exposure. The CVI of the questionnaire was 0.837 . The total Cronbach's score of the questionnaire was obtained through a pre-survey of 30 nursing students, $\alpha$ The coefficient is 0.890. Half a month later, the same group of nursing students were asked to fill in the same questionnaire, and the test-retest reliability coefficient of the questionnaire was 0.785 [4].

\subsection{Statistical analysis}

Spss20.0 statistical software was used for analysis, the measurement data was expressed with ( ), the comparison 
was performed with $t$ test, the repeated measurement data was analyzed with ANOVA, the count data was expressed with rate (\%), the comparison was performed with chi square test, $P<0.05$ was considered as statistically significant.

\section{Results}

\subsection{Comparison of knowledge, attitude and practice of occupational protection in chemotherapy be- tween two groups before and after internship}

The scores of knowledge, attitude and behavior of two groups of interns after practice were higher than those before practice $(P<0.05)$; after practice, the scores of knowledge, attitude and behavior of the system group were higher than those of the traditional group $(P<0.05)$ (see Table 1 ).

Table 1. Comparison of knowledge, attitude and practice of occupational protection in chemotherapy between two groups before and after internship (scores, $\bar{x} \pm s$ )

\begin{tabular}{cccccc}
\hline groups & $\mathrm{n}$ & time & knowledge & attitude & behavior \\
\hline system group & 50 & before practice & $12.97 \pm 0.38$ & $137.82 \pm 1.24$ & $150.54 \pm 1.02$ \\
& & after practice & $14.26 \pm 0.63^{\#^{*}}$ & $142.97 \pm 1.95^{\#^{*}}$ & $153.25 \pm 1.71^{\#^{*}}$ \\
traditional group & \multirow{2}{*}{50} & before practice & $12.96 \pm 0.40$ & $137.57 \pm 1.47$ & $150.87 \pm 0.95$ \\
& & after practice & $13.22 \pm 0.86^{\#}$ & $139.48 \pm 2.38^{\#}$ & $151.88 \pm 1.80^{\#}$ \\
\hline
\end{tabular}

Notes: compared with the same group before internship, ${ }^{\sharp} P<0.05$; Compared with the traditional group, ${ }^{*} P<0.05$.

\subsection{Comparison of occupational exposure to chemotherapy between two groups of interns before and after internship}

The occupational exposure scores of the two groups after internship were lower than those before internship ( $P<$ 0.05); After practice, the occupational exposure score of the system group was lower than that of the traditional group $(P<0.05)$ (see Table 2$)$.

Table 2. Comparison of occupational exposure to chemotherapy between two groups of interns before and after internship (scores, $\bar{x} \pm s$ )

\begin{tabular}{cccccc}
\hline groups & $\mathrm{n}$ & before practice & after practice & $t$ & $P$ \\
\hline system group & 50 & $68.71 \pm 7.68$ & $60.24 \pm 1.91^{\# *}$ & 6.965 & $<0.05$ \\
traditional group & 50 & $68.46 \pm 7.70$ & $63.04 \pm 1.24^{\#}$ & 4.541 & $<0.05$ \\
$t$ & 1.533 & 4.303 & & \\
$P$ & $>0.05$ & $<0.05$ & & \\
\hline
\end{tabular}

Notes: compared with the same group before internship, ${ }^{\#} P<0.05$; Compared with the traditional group, ${ }^{*} P<0.05$.

\section{Discussion}

There are essential differences between Green's health education model and general health education model. First of all, the general health education model only pays attention to the supplement of knowledge, but does not pay enough attention to the change of belief and the supervision of behavior; Green's health education model not only emphasizes knowledge supplement, but also emphasizes the intervention of belief and behavior to make the intervention more effective. Secondly, Green's health education model advocates systematic assessment of the characteristics and needs of groups and environment in advance before health education, and design a comprehensive health education intervention plan from the expected results, which can more effectively achieve the expected effect of changing individual behavior. Thirdly, the intervention process of Green's health education model is systematic, including nine consecutive steps: Sociological diagnosis, epidemiological diagnosis, behavioral environment diagnosis, management policy diagnosis, education ecological diagnosis, implementation, evaluation, impact evaluation and result evaluation. Evaluation is not only the overall evaluation of the implementation and intervention results, It also includes system evaluation before entering the next cycle framework [5]. Therefore, Green's health education intervention plan is more holistic and comprehensive, which ensures the effectiveness of the intervention and pro- 
vides us with new ideas, new perspectives and practical theoretical framework for health education intervention. In recent years, Green's health education model has been widely used in community health promotion and chronic disease intervention, public health, social science research and so on. However, it is rarely used in occupational protection of nursing interns in chemotherapy in China [6].

The data showed that the majority of nursing students had insufficient understanding of occupational injury, had fluke psychology, and could not standardize the implementation of protective measures [7]. Therefore, it is the key to cultivate good occupational protection behavior and reduce occupational injury for nursing students to correct the attitude of occupational protection, strengthen the awareness of protection, and clarify the important value of occupational protection to eliminate occupational injury. Relevant medical research shows that the application of green model to strengthen occupational protection education can improve nursing students' occupational protection cognition and attitude, and improve their occupational protection behavior [8]. The results showed that the scores of knowledge, attitude and behavior of two groups of interns after internship were higher than those before internship $(P<0.05)$; after practice, the scores of knowledge, attitude and behavior of the system group were higher than those of the traditional group $(P<0.05)$, which was consistent with the above research results. It is suggested that Green's health education mode is helpful to improve the occupational protection awareness of nursing students. The reason is that the continuous and dynamic supervision during the internship made the nursing students deeply realize the great harm of occupational exposure to themselves and the benefits of strict implementation of standard prevention, so as to correct the attitude of occupational protection. At the same time, green health education model improved the level of occupational protection knowledge of nursing students. The reason is that Green's health education model has strict framework guidelines, which is conducive to the researchers to implement health education for nursing students in a planned and targeted way. Secondly, the researchers use situational cases for assessment, which can guide nursing students to actively think about problems, which not only stimulates their interest in learning, but also changes the state of passive acceptance of knowledge in traditional health education, It is more helpful for nursing students to master occupational protection knowledge. Although the control group also received occupational protection health education lectures before internship, due to short learning time, incomplete content, and lack of continuous supervision during internship, it is difficult for the nursing students to form a systematic occupational protection knowledge structure, so the score of occupational protection knowledge of the control group is relatively low. In addition, green health education model enhanced the occupational protection behavior ability of nursing students. The reason is that health education intervention based on the framework of green model can improve the cognitive attitude and knowledge level of occupational protection of nursing students, so it is more conducive to master the protection skills, so as to further enhance the ability of occupational protection behavior. Although the control group also received the traditional occupational protection health education lectures before the internship, due to the lack of systematic and continuous health education intervention during the internship, the occupational protection behavior ability was relatively low.

It is reported that the causes of occupational exposure of nursing students in China include not only personal factors such as weak awareness of occupational protection, lack of knowledge and poor compliance, but also environmental factors such as lack of perfect supervision system and systematic occupational protection health education. Nearly $40 \%$ of nursing students had sharp injuries, and intensive education can reduce the incidence and improve the reporting rate [9]. Therefore, systematic, continuous and targeted occupational protection health education intervention is an effective way to reduce the occurrence of occupational exposure. The results showed that the occupational exposure scores of the two groups were lower than before $(P<0.05)$; after practice, the occupational exposure score of the system group was lower than that of the traditional group $(P<0.05)$. The reason is that the occupational protection health education under the framework of Green's health education model corrects the occupational protection attitude of nursing students, improves the protection awareness, and mobilizes the initiative to learn protection skills, so that nursing students pay more attention to the links prone to occupational exposure, thus effectively reducing the occurrence of occupational exposure.

Although individual occupational protection behavior mainly depends on individual, it has something to do with hospital and society. In the process of practice, the school and the hospital should jointly promote the formation of their occupational protection behavior. The school should establish the health files of nursing students and conduct regular physical examination to ensure their physical and mental health. The clinical nursing teachers should start with prevention, standardize management, strengthen occupational safety education of nursing students, take safe operation, set an example, reduce occupational hazards. Secondly, it is necessary to bring the occupational protection into the routine nursing operation, so as to improve the occupational protection awareness of nursing students. At the same time, the health administrative departments at all levels should constantly improve the protective laws 
and policies on occupational exposure, such as improving the emergency plan and handling procedures after the occurrence of hazards, taking a series of comprehensive preventive measures to create a safe working environment [10].

In conclusion, green education model has a good effect in improving the knowledge, attitude and practice of occupational protection in chemotherapy among nursing interns, which is worthy of promotion.

\section{References}

[1] Liu Jing, Zhou Xiaodong, and Cheng Linghong. (2021). The current situation of the nurses in the operating room on the smoke knowledge, faith and practice of operation and the intervention effect of green mode protection education [J]. Nursing Practice and Research, 2021, 18(4): 586-589.

[2] Wang Min, Li Yannan, li Yadong, et al. (2021). The influence of green health promotion model on the health cognition, compliance behavior and complication rate of glaucoma patients [J]. International Journal of Nursing, 2021, 40(9): $1603-1605$.

[3] Zhao Jing, Yu Meihua, Zhou Yan, et al. (2021). The effect of health management based on green model on nutritional status and prognosis of elderly patients with chronic heart failure [J]. Journal of Practical Cardio Cerebrovascular Disease, 2021, 29(1): 103-108.

[4] Du Jing, Wang Binquan, Ning Yan, et al. (2021). The effect of health education based on green model on improving the quality of life of sudden deafness patients [J]. Nursing Research, 2021, 35(5): 916-920.

[5] Zhu Qi, Zhou Jingyi, and Yuan Hongying. (2021). Effect of health education based on Green model on disease cognition, self-care behaviors and quality of life in patients with high risk of type 2 diabetes [J]. International Nursing Journal, 2021, 40(3): 476-480.

[6] Zhang Shimei, Xue Baomei, Li Na, et al. (2020). The application effect of nursing intervention based on green mode and wechat platform in patients discharged with T-tube after biliary tract operation [J]. Chinese Journal of Modern Nursing, 2020, 26(19): 2593-2597.

[7] Gao Zhanzhi. (2020). The influence of continuous rehabilitation nursing on hip function and self-care ability of hip joint in patients with hip fracture by wechat platform and green mode [J]. Chinese Medicine and Clinical, 2020, 20(1): 148-149.

[8] Weng Lingyi, Hu Huifang, Shi Xiangying, et al. (2020). The effect of health education on the abortion patients based on green model [J]. International Journal of Nursing, 2020, 39(1): 59-63.

[9] Mao Yanan. (2020). The value of prevention of blood-derived occupational exposure of nurses in operating room based on green model [J]. International Journal of Nursing, 2020, 39(10): 1743-1746.

[10] Jiang Yan, Zhang Huimin, Guo Lanqing, et al. (2019). Application of Green mode in health education for community women in breast cancer screening [J]. Nursing Research, 2019, 33(18): 3264-3266. 